\title{
Volasertib for AML: clinical use and patient consideration
}

This article was published in the following Dove Press journal:

OncoTargets and Therapy

17 July 2015

Number of times this article has been viewed

\section{Zhonglin $\mathrm{Hao}^{\prime}$ \\ Vamsi Kota ${ }^{2}$}

'Cancer Center, Medical College of Georgia, Georgia Regents University, Augusta, GA, USA; ${ }^{2}$ Winship Cancer Institute, Emory University School of Medicine, Atlanta, GA, USA
Correspondence: Zhonglin Hao

Cancer Center, Medical College of Georgia, Georgia Regents University, I4I Laney Walker Boulevard, Augusta, GA 30912, USA

Email zhao@gru.edu

\begin{abstract}
Acute myeloid leukemia (AML) is a disease diagnosed mostly in patients $>65$ years of age. Despite its heterogeneous nature, the different types of AMLs are still managed by standard induction chemotherapy for those who can tolerate it in the beginning. For the elderly and infirm patients, however, this approach leads to unacceptably high induction mortality rate. This article reviews past and current efforts searching for low-intensiveness treatments for the elderly and infirm patients who cannot tolerate the standard induction regimen. Volasertib, currently in Phase III clinical trials in combination with cytarabine, is reviewed as a promising agent for this patient population with AML, from the viewpoints of potential compliance and efficacy.
\end{abstract}

Keywords: volasertib, acute myeloid leukemia, management, induction

\section{Introduction}

Acute myeloid leukemia (AML) is a clonal hematological disorder characterized by proliferation of immature cells. This increased proliferation leads to cytopenias, which commonly present with neutropenia leading to infections, thrombocytopenia leading to bleeding complications, and anemia presenting as dyspnea on exertion or at rest. ${ }^{1}$ The incidence of AML increases with age, with the median age of diagnosis being $65-70$ years. $^{2}$ The cytopenias and the elderly age at presentation complicate the treatment with standard induction, showing very poor survival at 2 years. ${ }^{2}$ Even for patients of younger age ( $<60$ years), the standard-of-care treatments have not changed significantly for the better in the past. Anthracycline and cytarabine combination is still considered the first-line treatment. ${ }^{3}$ This is one of the main reasons for the continued investigation of newer drugs in the treatment of AML. This article covers approaches to the elderly and the infirm patients with AML and reviews in depth the new cell cycle inhibitor volasertib, its mechanism of action, pharmacology, and its promise in becoming the standard of care for elderly and infirm patients with AML.

\section{Risk stratification and current management of AML}

Chromosomal evaluation has long played an important role in risk stratification of AML patients. ${ }^{4}$ On the basis of whether patients have abnormal cytogenetics or according to the types of abnormal cytogenetics in the leukemia blasts, AML is stratified into four different risk groups: Favorable, Intermediate I, Intermediate II, and Adverse risk groups. ${ }^{4}$

Induction therapies are similar in all patients. However, postremission care may vary, with allogeneic stem cell transplant being the preferred option in poor and some intermediate risk groups. The favorable-risk AML patients generally are expected to do well with postremission consolidation chemotherapy alone. ${ }^{5,6}$ Acute promyelocytic 
leukemia, which is characterized by translocation (15:17) in most patients and variant translocation involving chromosome 17 in others, is a unique subset and is treated differently. All-trans retinoic acid and arsenic trioxide have been shown to be very effective as frontline treatments in patients presenting with white cell counts $<10,000 / \mathrm{mL}$. This subset of AML can be considered one of the first cancers for which targeted therapy has been used successfully. Cure has been achieved in a significant proportion of patients in this group. Identification of this subset has allowed us to tailor the treatments to this type of AML and laid the foundation for our approach to AML, which is increasingly recognized, genetically, as a very heterogeneous disease.

\section{Genetics and epigenetics in AML: understanding the heterogeneity of AML}

Cytogenetic evaluation has been the cornerstone of risk stratification, but $50 \%$ of patients have "normal cytogenetics", making this one the largest subsets of all AML patients. ${ }^{7,8}$ Further classification of these patients has been increasingly pursued both for prognostic reasons and for better identification of patients who will benefit from targeted therapies. ${ }^{9}$ The advent of molecular diagnostics has increased our understanding of the genetic heterogeneity of this disease. The most recent European LeukemiaNet (ELN) guidelines now incorporate the mutational status of internal tandem duplications (ITDs) in FLT3 (encoding fms-related tyrosine kinase 3), CEBPA (encoding CCAAT/enhancer binding protein $(\mathrm{C} / \mathrm{EBP})$, alpha), and NPM1 (encoding nucleophosmin) into risk stratification considerations, which allows us to better direct care specifically to the intermediate-risk group for AML. ${ }^{3}$ Other than identifying the driver mutations of disease, another advantage of identifying these mutations might be in the monitoring of minimal residual disease (MRD). Although it has not been validated for all mutations yet, NPM1 mutation has been shown to be useful in monitoring MRD. ${ }^{10}$

NPM1 is the most common mutation detected in AML. It is found in $25 \%-30 \%$ of all AML patients and in $45 \%-50 \%$ of patients who are cytogenetically normal $(\mathrm{CN}) .{ }^{11}$ This mutation has been well documented to confer chemosensitivity and is generally considered to be a good prognostic marker. ${ }^{12}$ Although it is widely accepted to be a valid good prognostic marker in all age groups, some emerging data suggest that in patients older than 65 years of age, this mutation might not have as good outcomes. ${ }^{13}$ If confirmed, alternative treatment practices might need to be considered even with this mutation. Present practice withholds allogeneic transplant in patients with this mutation regardless of age. The concurrent presence of the FLT3-ITD mutation also leads to poor outcomes in NPM1-mutated patients.

FLT3-ITD mutations are present in approximately onethird of all CN-AML patients. Presence of such mutations is widely accepted to confer poor outcome in AML. The allelic burden of this mutation seems to be important prognostically as well, with high allelic burden having worse outcomes. However, the importance of lower burden is still a matter of controversy. ${ }^{9}$ A different insertion point at the tyrosine kinase domain, hence labeled as FLT3-TKD, also confers a worse outcome. ${ }^{14}$ Multiple drugs are being investigated as potential inhibitors of FLT3. So far, however, the results have been with varying success. ${ }^{15}$

$C E B P A$ is a less common mutation as compared to NPM1 and FLT3-ITD. It confers better prognosis, although only double mutations can be considered significant. This mutation is also commonly seen in association with NPM1 or FLT3-ITD. The outcome might be different when it is associated with FLT3-ITD. ${ }^{9}$

DNMT3A [encoding DNA (cytosine-5-)-methyltransferase 3 alpha] mutations are seen in approximately one-third of all CN-AML patients. ${ }^{16}$ These mutations appear to confer worse outcomes. There are, however, some conflicting studies on the effects in younger patients. ${ }^{16,17}$ In a recent study, younger patients who had this mutation appeared to have benefited from increasing doses of anthracycline. ${ }^{18}$

IDH1 and IDH2 (isocitrate dehydrogenases 1 and 2) are mutated in approximately $25 \%$ of CN-AML patients. Although the prognostic significance is still debated, ${ }^{18}$ availability of targeted therapy inhibiting these mutations might allow us to better treat these patients if ongoing studies show a favorable outcome. ${ }^{19,20}$

TET2 (tet methylcytosine dioxygenase 2) and ASXL1 mutations are less common mutations in AML. Both appear to be more common with increasing age among AML patients. ASXL1 mutations appear to confer a poor outcome, ${ }^{9}$ and the prognostic significance of the TET2 mutation is still controversial, with studies showing conflicting results on overall survival (OS)..$^{21,22}$

\section{Standard-of-care treatment}

The standard-of-care treatment for AML includes an upfront induction therapy, followed by a risk-adapted consolidation therapy. Induction chemotherapy for AML patients who can tolerate intensive treatment generally consists of combinations of an anthracycline with cytarabine. Various trials have 
been conducted to add additional drugs to this combination, but none of them showed any significant benefit to change the clinical practice. ${ }^{23}$ Studies conducted on changing the dose of cytarabine or anthracycline (daunorubicin versus idarubicin) did not show any consistent benefit. As a result, a single regimen has become the standard of care that is widely used. ${ }^{24,25}$ A recent study comparing daunorubicin $90 \mathrm{mg} / \mathrm{m}^{2}$ to $45 \mathrm{mg} / \mathrm{m}^{2}$ in patients younger than 60 years of age showed the superiority of the higher dose, and this regimen is increasingly being used in the community. ${ }^{26} \mathrm{~A}$ similar study comparing a higher dose to the conventional dose of daunorubicin showed that the higher dose achieved better responses in elderly patients. ${ }^{27}$ The goal of induction treatments is to achieve a complete remission (CR). The latter is traditionally defined as bone marrow blasts $<5 \%$ and recovery in blood counts with absolute neutrophil count of $>1,000 / \mathrm{mL}$ and platelets $>100,000 / \mathrm{mL}$. The rate of CR in younger patients with the high-dose daunorubicin/cytarabine combination approaches $70 \%$ after one cycle of induction as compared to $50 \%-60 \%$ $\mathrm{CR}$ rates with the standard dose of daunorubicin.

Many more trials were conducted with varying doses of cytarabine. However, 100-200 mg/ $\mathrm{m}^{2}$ of cytarabine daily for 7 days as continuous infusion is considered the conventional dose along with daunorubicin or idarubicin for the first 3 days. Higher doses of cytarabine have been tested. Although some studies have suggested relapse-free survival (RFS) benefit, large randomized studies have not shown an OS advantage. The common practice is still a conventional dose of cytarabine for induction in both the young- and the older-aged patients. ${ }^{28}$

\section{Older adults}

The induction treatment of AML in elderly patients, which generally refers to those older than 65 years of age, is much more complicated, although this is the more commonly seen patient population with this disease. In patients who can tolerate intensive chemotherapy, induction with highdose daunorubicin and cytarabine has been shown to be beneficial in achieving CR. Unfortunately, most patients are not eligible for induction therapy due to age and performance status. Large cooperative group studies carried out by the Eastern Cooperative Oncology Group (ECOG), the Southwest Oncology Group (SWOG), and the UK National Cancer Research Institute (NCRI) showed that the probability of 2-year survival in elderly patients undergoing intensive treatment is only approximately $20 \%-25 \%$, with CR rates after induction of approximately $40 \%-50 \%$. The high rate of induction mortality $(15 \%-19 \%)$ might be one reason for the poor outcomes. These survival data again included only patients who were considered to be well enough to receive intensive treatments. ${ }^{29-31}$ Various prognostic models are being developed to help us choose patients for induction therapy as induction therapy still offers the best chance of achieving remission in patients who can tolerate it. ${ }^{32,33}$

In addition, it is well known that older age has been associated with a decrease in the number of patients with favorable cytogenetics, from $30 \%$ to only $7 \%$, and doubling of complex cytogenetics, from $6 \%$ to $12 \%$. As a result, the 5 -year survival is only $13 \%$ compared with $57 \%$ in the youngest age group. ${ }^{34}$ This means that therapeutic agents such as volasertib that work across different cytogenetic risk groups are expected to offer an advantage in the treatment of AML in elderly patients because current agents are frequently ineffective in the complex cytogenetic group (refer section on "Consolidation therapy").

\section{Consolidation therapy}

In patients who achieve $\mathrm{CR}$ after induction therapy, lack of additional treatments invariably leads to relapse of disease in almost all patients. ${ }^{35}$ Ever since the Cancer and Leukemia Group B presented the landmark results in 1994 showing superiority of high-dose cytarabine $\left(3 \mathrm{~g} / \mathrm{m}^{2}\right)$ versus intermediate dose $\left(400 \mathrm{mg} / \mathrm{m}^{2}\right)$ versus low dose $\left(100 \mathrm{mg} / \mathrm{m}^{2}\right)$, it has become the standard of care in most practices. This high dose is given every 12 hours on Days 1,3 , and $5 .{ }^{36}$ Newer studies with lower doses $\left(1.5 \mathrm{~g} / \mathrm{m}^{2}\right)$ have shown similar benefit with less toxicity. Therefore, lower doses can also be used in consolidation treatment. The number of cycles is not clearly established, although it is common to give three to four cycles. ${ }^{37}$

Consolidation therapy in elderly patients who tolerated induction and are eligible to receive intensive therapy again is probably best limited to one to two cycles of consolidation, with $1.0-1.5 \mathrm{~g} / \mathrm{m}^{2}$ of cytarabine over 4-6 days in each cycle. Another option can be as per the recent Acute Leukemia French Association (ALFA) 98 trial documenting the superiority of the ambulatory arm. This regimen consisted of an anthracycline (idarubicin $90 \mathrm{mg} / \mathrm{m}^{2}$ or daunorubicin $45 \mathrm{mg} / \mathrm{m}^{2}$ ) for 1 day and $60 \mathrm{mg} / \mathrm{m}^{2}$ of cytarabine every 12 hours for 5 days each month for 6 months. ${ }^{38}$

Postremission management should include discussions of allogeneic stem cell transplant in all patients with intermediate or poor risk of disease, classified as such per the new ELN guidelines. Age alone need not be an exclusion criterion, with most transplant centers routinely considering transplants up to the age of 70 years. The Hematopoietic Cell Transplantation Comorbidity Index has been shown 
to be a good predictor of outcomes based on pretransplant comorbidities. ${ }^{39}$

\section{Pitfalls of standard chemotherapy}

As noted earlier, AML is a disease of the elderly population, with more than half of all patients being above 65 years of age. Unfortunately, the disease biology appears to be different in the elderly, with higher chance of antecedent myelodysplastic syndromes (MDS), higher chance of multidrug resistance, and poor performance status. An evaluation of patients enrolled under the SWOG trials showed that patients older than 66 years of age had a $20 \%$ chance of mortality within 30 days, and this mortality rate was even higher for patients over the age of 75 years. This high mortality was seen even in patients with good performance status. ${ }^{40}$ Therefore, alternative low-intensiveness therapies were vigorously sought.

It is also widely recognized that patients enrolled in clinical trials comprise a selected group of patients, and it is likely that the outcomes would be worse if the same intensive treatments were applied to those in the general population. Patients treated on a clinical trial are likely to be given curative intent treatment and their outcomes are likely to be better. This might not be extrapolated to the general population as it is likely that patients in that age group have comorbid conditions. These patients with comorbid conditions are frequently excluded from clinical trials. ${ }^{41}$ A recent Surveillance, Epidemiology and End Results (SEER) and Medicare review of outcomes of patients with AML showed that $60 \%$ of patients are not offered any of the standard recommended options of chemotherapy, including hypomethylating therapy. ${ }^{42}$ Thus, the availability of newer agents that are better tolerated might change this approach in clinical practice even in the elderly or in patients with comorbid conditions who are routinely deemed unfit for any treatments. At least four options are currently available or are being studied, as described in the following sections.

\section{Low-dose Ara-C}

For the elderly and infirm patients deemed unable to tolerate the standard induction chemotherapy with an anthracycline and cytarabine combination, low-dose cytarabine (low-dose Ara-C or LDAC) is better than supportive care. This was shown in a Phase III trial in 2007 comparing LDAC and hydroxyurea (HU). ${ }^{43}$ In this trial, LDAC offered $18 \% \mathrm{CR}$ rate compared to only $1 \%$ in the $\mathrm{HU}$ arm $(P=0.00006)$. Most importantly, those who received LDAC had OS advantage relative to $\mathrm{HU}$ recipients (hazard ratio [HR]: 0.60; 95\% confidence interval $[\mathrm{CI}]: 0.44-0.81 ; P=0.0009)$. LDAC is commonly used for this group of patients.

\section{Hypomethylating agents in AML}

Azacitidine and decitabine are approved in the USA for the treatment of MDS. Decitabine is also approved in the European Union for the newly diagnosed de novo or secondary AML in patients aged $>65$ years or for those who cannot tolerate standard induction chemotherapy. These agents are well tolerated, and the response rates are only slightly better as compared to the best supportive care plus low-dose cytarabine in most patients. Patients with low blast counts in the range of $20 \%-30 \%$ appear to benefit more from these agents as compared to patients with a very high disease burden. These are good results as compared to available chemotherapy options. In addition, patients with complex cytogenetics and TP53 mutations are especially suited for such option given their poor response to conventional chemotherapy. ${ }^{44}$ A Phase III study compared decitabine with supportive care or LDAC in patients aged 65 years or older with newly diagnosed AML in the poor- or intermediaterisk category. ${ }^{45}$ The $\mathrm{CR}$ and $\mathrm{CR}$ without platelet recovery (CRp) were $17.8 \%$ versus $7.8 \%$ (odds ratio [OR]: $2.5 ; 95 \%$ CI: $1.4-4.8 ; P=0.001)$. Although there was no difference in OS per protocol analysis (7.7 months versus 5.0 months; HR: 0.85 ; 95\% CI: 0.69-1.04; $P=0.108)$, unplanned analysis based on mature survival data did show significant OS benefit (HR: 0.82; 95\% CI: $0.68-0.99 ; P=0.037$ ). The most common adverse effects were thrombocytopenia (27\%) and neutropenia (24\%). The US Food and Drug Administration did not approve the supplementary data. Results of the Phase III trials are pending. The end point for the azacitidine Phase III trial is OS. For decitabine, they are CR, OS, event-free survival (EFS), and RFS. ${ }^{46}$

\section{Gemtuzumab ozogamicin}

Gemtuzumab ozogamicin (GO) is a monoclonal antibody directed against the CD33 cell surface antigen expressed on AML cells, conjugated to an antibiotic calicheamicin, which causes DNA strand break and cell death on internalization. GO was approved in the USA and Japan for the treatment of relapsed AML patients aged older than 60 years and ineligible for standard induction chemotherapy. GO was however subsequently withdrawn from the market due to increased risk of death during induction in a trial conducted after approval. ${ }^{47}$ The Phase III trial of GO alone in comparison with the best supportive care is pending results. This trial targets patients previously untreated but not eligible for intensive chemotherapy (NCT00091234).

In the Phase II monotherapy trial, ${ }^{48} \mathrm{CR}, \mathrm{CRp}$, and PR (partial response) were achieved in $23 \%$ of patients in both 
treatment schedules, with an early mortality rate of $12 \%$. Adverse effects included nausea, vomiting, diarrhea, stomatitis, and transient elevation in liver function tests.

The LRF AML14 and NCRI AML16 trials compared GO plus LDAC with LDAC alone. ${ }^{49}$ GO plus LDAC improved the response rate compared to LDAC alone (30\% versus $17 \%$; HR: $0.48 ; 95 \%$ CI: $0.32-0.73 ; P=0.006)$. The $12-$ month OSs were not different ( $27 \%$ versus $25 \%$; HR: $0.99 ; 95 \% \mathrm{CI}$ : $0.83-1.16 ; P=0.9)$. The 30 -day mortalities were not different (18\% versus $16 \%$ ).

\section{Cell cycle inhibitor volasertib}

The Polo-like kinases (Plks) consist of a family of five serine-and-threonine kinases, with four being identified in mammals. ${ }^{50-52}$ The first polo gene was isolated in 1991 by a group working with the fruit fly Drosophila melanogaster. ${ }^{53}$ It was found that this gene encodes an enzyme, mutation of which was associated with aberrant mitosis. Subsequent work $^{54,55}$ identified the murine and human homologs of the fly gene, involved in cell proliferation. They were mainly expressed in the hematopoietic progenitor cells, testes, and ovaries in contrast to other tissues with low proliferation rate. They were named polo-like kinases, Plks. The kinases were homologous to the budding yeast Saccharomyces Cerevisiae cell division cycle gene $C D C 5$ and early growth response gene $S N K / P L K 2$; all belong to the big family of serine-and-threonine kinases. The following section will review efforts in bringing the $P l k l$ inhibitor volasertib to the bedside for AML patients who cannot tolerate standard induction chemotherapy.

\section{PLKI gene structure and function}

Structurally, PLKs have two functional domains: the N-terminal serine/threonine kinase catalytic domain and the C-terminal regulatory domain(s). ${ }^{56,57}$ The catalytic domain is where the serine/threonine kinase activity resides and has an adenosine triphosphate (ATP)-binding pocket, to which the small-molecule ATP-competitive kinase inhibitors are directed. The regulatory domain consists of one to two polo-box domains (PBDs). The $\mathrm{PBD}(\mathrm{s})$ inhibit the catalytic domain when there is no binding of phosphopeptide. On binding to the phosphopeptide of a phosphoprotein, the PBD releases the kinase domain, allowing it to bind or dock its substrate, thus setting off the signal transduction cascade. The PLKs play essential roles in many important cellular processes. These processes include mitosis, ${ }^{58}$ DNA replication, ${ }^{59}$ and stress response to DNA damage and recovery, ${ }^{60-62}$ among other newly discovered ones such as resistance to apoptosis ${ }^{63,64}$ and regulation of cancer cell invasiveness. ${ }^{65}$ The role of PLK1 is the best studied. Its essential roles in mitosis include mitotic entry, centrosomal maturation, sister chromatid separation, mitotic exit, as well as cytokinesis (Figure 1). The most prominent phenotype occurring when PLK1 function is depleted is known as the "polo-arrest" (Figure 2) ${ }^{66}$ When polo-arrest happens, there is perturbation of the spindle assembly and cell cycle arrest at the prometaphase. Mitotic cells accumulate with monopolar spindles that are inappropriately attached to the kinetochores. In the end, cells go into apoptosis. ${ }^{67}$

\section{PLKI as a valid target in cancer therapy}

As stated herein, PLK1 is expressed in normal proliferating cells. PLK1 is also frequently overexpressed in various malignancies such as AML, non-small cell lung cancer (NSCLC), breast cancer, prostate cancer, colorectal cancer, pancreatic cancer, papillary thyroid cancer, ovarian cancer, head and neck cancer, as well as in non-Hodgkin's lymphomas. ${ }^{68-71}$ Studies have also linked PLK1 overexpression to poor prognosis and survival. Therefore, it has long been considered a valid target for cancer therapy.

\section{Volasertib is a small-molecule Plk I inhibitor}

Volasertib (BI6727) belongs to the class of dihydropteridinone derivative small-molecule ATP-competitive kinase inhibitors. ${ }^{66}$ Volasertib was discovered by screening a library of organic compounds capable of inhibiting Plk1 activity. Although it is of the same class with BI2536 $6^{67,72}$ (also a very potent, small-molecule dihydropteridinone derivative ATP-competitive kinase inhibitor), volasertib has a different chemical structure and was tailored from BI2536 based on the principles of potency, selectivity, and efficacy in inhibiting tumor growth in the xenograft tumor model.

\section{Pharmacology of volasertib}

Volasertib is potent and selective. It has a half-maximal inhibitory concentration $\left(\mathrm{IC}_{50}\right)$ of $0.87 \mathrm{nM}, 5.0 \mathrm{nM}$, and $56 \mathrm{nM}$ toward Plk1, Plk2, and Plk3, respectively. ${ }^{66}$ Volasertib had no appreciable inhibition to a panel of $>60$ other kinases tested at a concentration as high as $10 \mathrm{M},{ }^{73}$ asserting the high selectivity of the drug. The half-maximal effective concentration $\left(\mathrm{EC}_{50}\right)$, which measures the potency of a drug in a battery of cell lines, was between $11 \mathrm{nM}$ and $37 \mathrm{nM}$. Cell lines used in determining the $\mathrm{EC}_{50}$ included lung cancer (H460), colon (HCT116), melanoma (BRO), leukemia (HL60), and B-cell lymphoma (Raji). The half-life $\left(T_{1 / 2}\right)$, when tested in humans, 


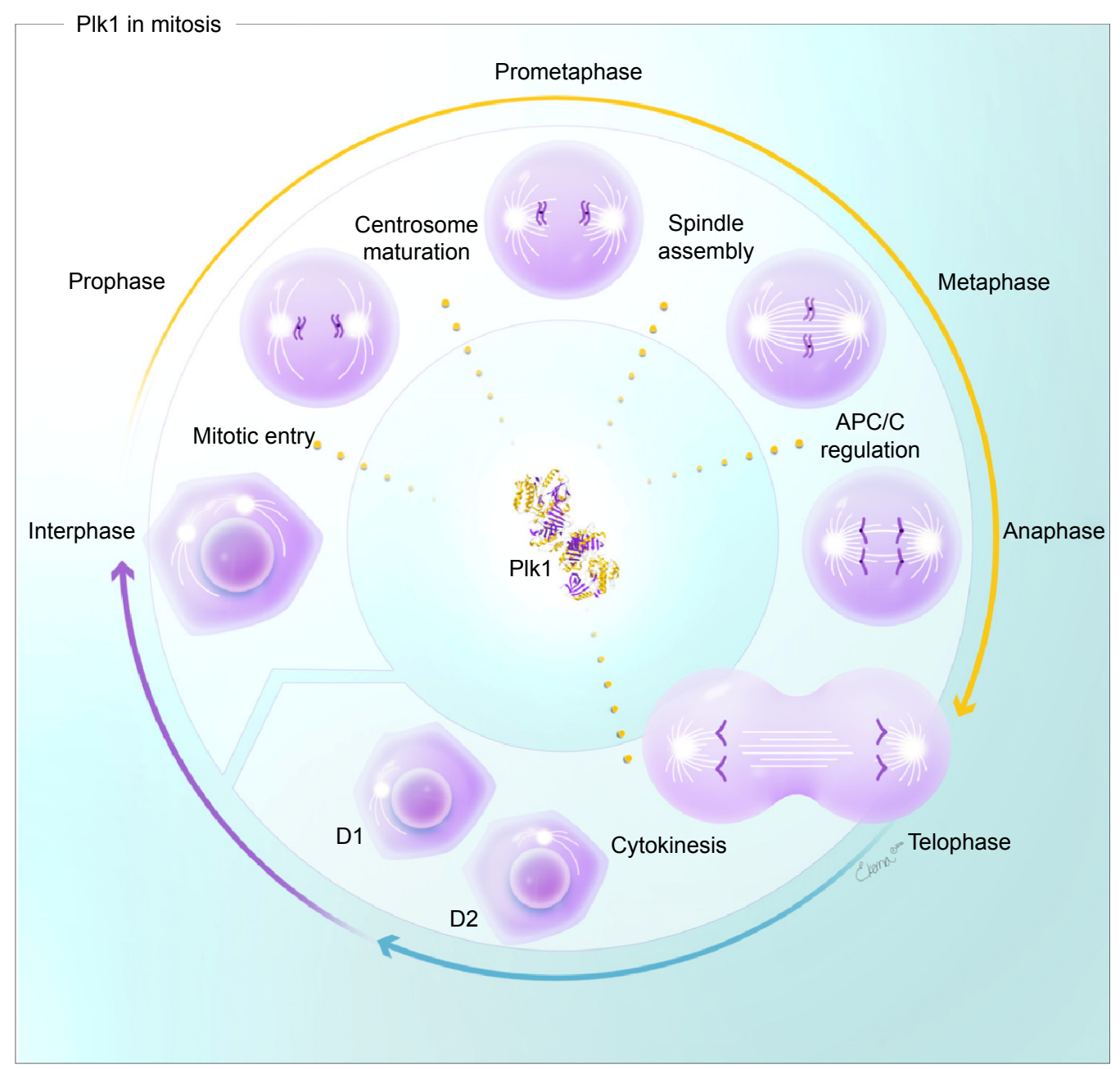

Figure I Functions of PlkI during the cell cycle.

Notes: Dashed lines indicate the sites of action. Courtesy of Lynsey Ekema, MSMI Instructional Design and Development, Georgia Regents University. Abbreviations: APC/C, anaphase-promoting complex/cyclosome; D, daughter cell; Plk, Polo-like kinase.
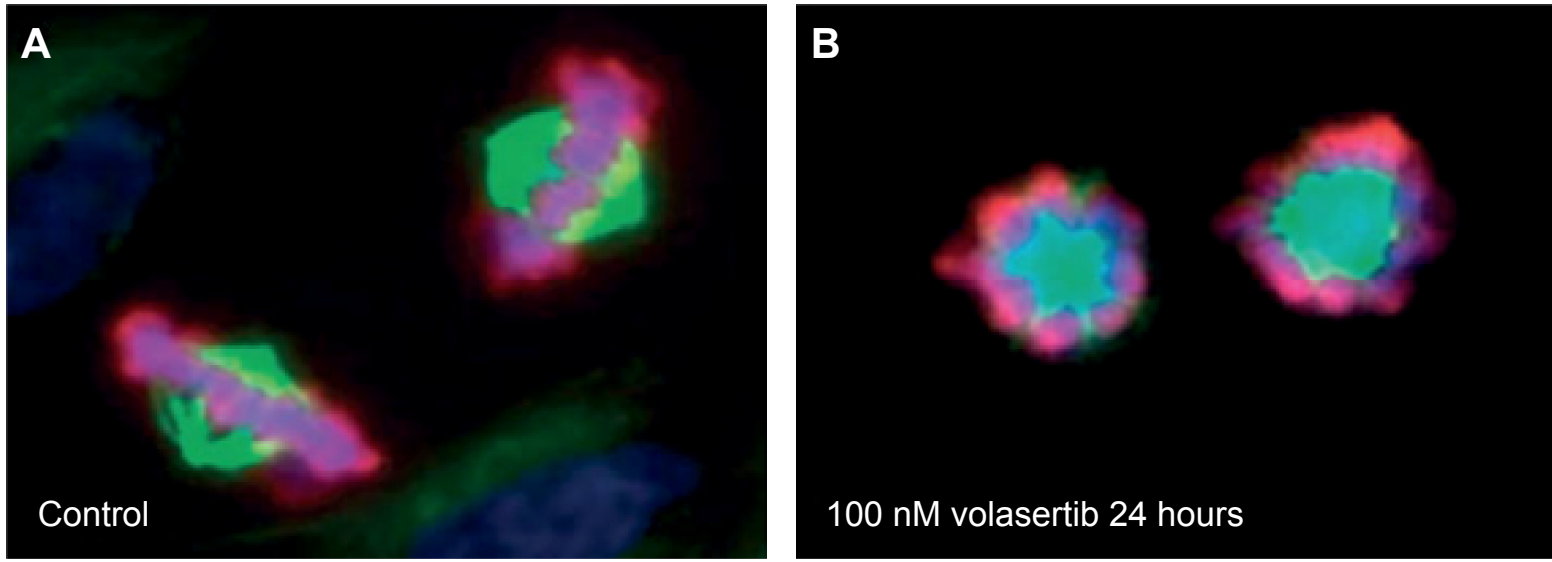

Figure 2 Volasertib blocks bipolar spindle formation, inducing cell cycle arrest in the $M$ phase.

Notes: NCl-H460 NSCLC cells were treated for 24 hours with either (A) $0.1 \%$ DMSO or (B) $100 \mathrm{nM} / \mathrm{L}$ of volasertib. Cells were fixed, stained with either DAPI (to stain DNA; in blue) or anti-tubulin (to stain spindles; in green) and anti-phosphoSerl0 histone $\mathrm{H} 3$ (in pink), and photographs taken under a fluorescence microscope. Volasertib treatment caused accumulation of mitotic cells with monopolar spindles. The kinetochores here were not properly attached to the spindle, ie, they showed "Polo arrest." Reprinted from American Association for Cancer Research, 2009, 15/9, 3094-3102, Dorothea Rudolph, Martin Steegmaier, Matthias Hoffmann et al., BI 6727, A Polo-like Kinase Inhibitor with Improved Pharmacokinetic Profile and Broad Antitumor Activity, with permission from AACR. ${ }^{66}$

Abbreviations: AACR, American Association for Cancer Research; DAPI, 4',6-diamidino-2-phenylindole; DMSO, dimethyl sulfoxide; NCl, National Cancer Institute; NSCLC, non-small cell lung cancer. 
was 111 hours, with a volume of distribution of $>4,000 \mathrm{~L}$. The clearance was moderate, at $792 \mathrm{~mL} / \mathrm{min}$. All these pharmacokinetic characteristics suggest very good tissue exposure to volasertib. The long half-life and large volume of distribution were also confirmed in an independent study in AML patients. ${ }^{74}$ The oral bioavailability is good across species: mouse ( $F: 41 \%)$, rat (F: $55 \%)$, and $\operatorname{dog}(F: 53 \%)$.

\section{Tolerability and safety}

In the first-in-human study, volasertib was given in a dose escalation manner in a Phase I trial that treated 65 patients with progressive metastatic solid tumors. ${ }^{75}$ Of these, 51 patients were treated during the dose escalation phase and 14 others in the expansion cohort. Patients received a single 1-hour infusion of volasertib every 3 weeks $(\mathrm{Q} 3 \mathrm{~W})$. The doses received ranged from $12 \mathrm{mg}$ to $450 \mathrm{mg}$. Side effects were all mainly hematological and were reversible. Dose-limiting toxicity (DLT) started to appear when the dose was escalated to $300 \mathrm{mg}$. DLT mainly included neutropenia, thrombocytopenia, and febrile neutropenia. The most common drug-related adverse events were anemia (total: $22 \%$; grade $3: 8 \%$ ), neutropenia (total: $15 \%$; grade $3 / 4: 14 \%$ ), thrombocytopenia (total: $14 \%$, grade $3 / 4: 14 \%$ ), and fatigue (total: $15 \%$, grade $3: 2 \%$ ). The maximum tolerated dose (MTD) was initially determined to be $400 \mathrm{mg}$ Q3W. However, during the expansion cohort, three out of ten patients had DLT. After dose de-escalation to $350 \mathrm{mg}$, there were again three out of five DLTs. Therefore, the recommended dose for Phase II was $300 \mathrm{mg}$. Fatigue, weight loss, and QT prolongation were the other DLTs encountered in the trial. Volasertib was well tolerated.

In another confirmatory Phase I study conducted in 59 Asian patients, ${ }^{76}$ DLTs were again thrombocytopenia, neutropenia, and febrile neutropenia. The MTD determined for the Q3W infusion was $300 \mathrm{mg}$ and $150 \mathrm{mg}$ for the Day 1/Day 8 Q3W schedule. Pharmacokinetic characteristics again showed a long $T_{1 / 2}$ of 135 hours, with a large volume distribution of $>3,000 \mathrm{~L}$ and moderate clearance. Volasertib was again well tolerated.

In a Phase I ${ }^{74}$ study of volasertib proceeding the Phase II trial $^{74}$ in combination with low-dose cytarabine (LDAC) in relapsed/refractory AML, volasertib was given in a dose escalation manner on Day 1 and Day 15 every 4 weeks (Q4W) alone or in combination with subcutaneous LDAC $20 \mathrm{mg}$ bid on Days 1-10 Q4W in a dose de-escalation fashion. In the monotherapy arm, 29 patients (age range: $26-84$ years, median: 71 years) were evaluated. Adverse effects were reported in eight patients, which were considered drug related $(27.6 \%)$. The most common drug-related adverse effects were anemia (10.3\%), thrombocytopenia, epitaxis, and nausea (6.9\%). Grade 3/4 drug-related adverse effects included thrombocytopenia, anemia, diarrhea, mucositis, pneumonia, fungal pneumonia (grade 5), and neutropenia. DLTs were grade 4 pneumonia, fatal fungal pneumonia that arose in a patient receiving $150 \mathrm{mg}$ of volasetib, and one case of grade 3 mucositis (at $400 \mathrm{mg}$ ). MTD was not reached at $500 \mathrm{mg}$. In the combination arm, MTD was determined to be $350 \mathrm{mg}$ at Day 1 and Day 15 Q4W, with Ara-C given at $20 \mathrm{mg}$ bid, subcutaneously, on Days 1-10. No drug interaction was observed after administration of Ara-C with volasertib. The combination with Ara-C is safe and well tolerated too. This conclusion was supported by further treatment of AML in the Phase II part of the study. ${ }^{74}$

\section{Efficacy of volasertib}

\section{Preclinical evaluation in animal models}

The efficacy of volasertib was first observed in human tumor xenograft models established from multiple human cancer cell lines (colon: HCT116, lung: H460, and taxane-resistant colon: CXB1) in nude mice. ${ }^{66}$ Multiple rounds of volasertib were given to animals at a frequency ranging from daily to once or twice a week either orally or intravenously for 6 weeks. In the colon cancer model, volasertib had similar efficacy at a total weekly dosage of $50 \mathrm{mg} / \mathrm{kg}$. Similar results were obtained when animals received $20 \mathrm{mg} / \mathrm{kg}$ or $30 \mathrm{mg} / \mathrm{kg}$ of volasertib intravenously once a week whereas tumors in the control group were progressively larger, volasertib given at $20 \mathrm{mg} / \mathrm{kg}$ for two consecutive days per week for 5 cycles resulted in tumor regression. Volasertib delayed tumor growth in NSCLC. Furthermore, it was effective in inhibiting the taxane-resistant colon cancer model as well. Examination of the tumor tissue after volasertib treatment found a 13 -fold increase in mitotic figures in the tumor compared to the control in a colon cancer model 24 hours later. Apoptosis, as demonstrated by the terminal deoxynucleotidyl transferase deoxyuridine triphosphate nick-end labeling (TUNEL) assay, increased 4.5 folds compared to tumor treated with vehicle only. When a mouse bearing the colon cancer was given a single dose of $35 \mathrm{mg} /$ $\mathrm{kg}$, a much higher tissue concentration of volasertib is reached (maximum of $32 \mu \mathrm{M} 8$ hours versus $7 \mu \mathrm{M} 1$ hour after administration). Volasertib concentration in the tissue was still $4 \mu \mathrm{M}$ in the tissue compared to only $8 \mathrm{nM}$ in the blood 168 hours (7 days) after the drug injection (500-fold difference).

\section{Phase I studies in solid tumors}

In the first Phase I study, ${ }^{75}$ three patients showed an objective response, all of them PR, by Response Evaluation Criteria 
In Solid Tumors (RECIST) criteria. Types of solid tumors included melanoma (12), NSCLC (10), colorectal cancer (8), soft tissue sarcoma (7), urothelial carcinoma (6), prostate cancer (4), and others (18). The melanoma patient who showed PR was administered the $300 \mathrm{mg}$ once Q3Wdosage. The patient had received cisplatin and dacarbazine followed by radiation therapy, before failure of ipilumumab occurred. The PR started from Cycle 2 and lasted till Cycle 9 (progressionfree survival [PFS]: 207 days). One patient with urothelial cancer had PR from Cycle 2 to Cycle 16. This patient had a dose reduction to $300 \mathrm{mg}$ from Cycle 2 onward after adverse effect was noted on getting $450 \mathrm{mg}$ in Cycle 1. The patient was restarted on volasertib after tumor resection and received a total of 39 cycles; PFS was 403 days. This patient was also heavily treated with neoadjuvant gemcitabine/cisplatin with surgery on diagnosis, and then paclitaxel, capecitabine with investigational agent after recurrence. The third patient with PR was administered $300 \mathrm{mg}$ of drug (decreased after the first course from $400 \mathrm{mg}$ ) for her ovarian cancer. She had a total of four cycles and PFS of 148 days. After recurrence, this patient received six cycles of carboplatin/paclitaxel with $\mathrm{CR}$, four cycles of cisplatin intraperitoneally, six cycles of liposomal doxorubicin, letrozole, and topotecan before volasertib. It is also noted in this trial that one NSCLC patient at $300 \mathrm{mg}$ dose had stable disease as the best response for 550 days. This patient had no response to cisplatin-based chemotherapy and then to taxotere, with progressive disease in both occasions. Forty percent of these patients had stable disease as the best overall response and $48 \%$ had clinical benefit.

In a separate Phase I study performed in 59 Asian patients, ${ }^{76}$ two more PRs were documented. One had urothelial carcinoma receiving $300 \mathrm{mg}$ Q3Wand another had melanoma receiving $150 \mathrm{mg}$ at Day 1 and Day 8 . The urothelial cancer patient received a total of 23 cycles, whereas the melanoma patient received 9 cycles. Stable disease was found in $44.1 \%$ patients as their best response.

\section{Phase I/II studies in AML patients}

In the Phase I part of the study, antileukemic activity was observed in patients who had received higher doses of $\geq 350 \mathrm{mg}$ intravenously on Day 1 and Day 15 Q4W in the monotherapy arm. CR or CR with incomplete blood count recovery (CRi) was achieved in 4 of the 16 patients. In the combination arm with LDAC, 7 out of 32 patients treated achieved CR or CRi. Median OS was 551 days (range: 165-595 days). ${ }^{74}$

In the randomized Phase II part comparing LDAC versus volasertib plus LDAC, 87 patients were treated in two arms.
The response rate (CR and $\mathrm{CRi}$ ) in the LDAC plus volasertib arm was 31\% (13 of 42 patients) compared to $13.3 \%$ in the LDAC arm (6 out of 45 patients) (OR: 2.91, $P=0.052$ ). There was no apparent correlation between the white blood cell count or blast percentage in the bone marrow at presentation. Importantly, the response was observed across all cytogenetic groups (regardless of the group as per ELN classification, Wheatley risk group, or genetic mutations in FLT3-ITD, NPM1, as described for different risk groups of leukemia). The patients in the combination arm had significantly longer exposure to the study drug than the LDAC arm (309 days versus 214 days). ${ }^{74}$

At the time of survival analysis, 77 of the 87 patients had died. EFS in patients receiving the LDAC and volasertib $(n=42)$ was significantly longer than in patients receiving LDAC only $(\mathrm{n}=45)$ (5.6 months versus 2.3 months; HR: 0.57 ; 95\% CI: $0.35-0.92 ; P=0.021)$. RFS was 18.5 months and 10.0 months for the combination group $(n=13)$ and the LDAC-only group $(n=6)$, respectively, suggesting longer duration of remission in the combination treatment group. The median OS for the combination group and the LDAC group were 8.0 months and 5.2 months, respectively (HR: 0.63; 95\% CI: 0.4-1.00; $P=0.047)$. Exploratory analyses comparing survival of patients in the same cytogenetic groups treated in the two arms showed benefit in adding volasertib. Of note, this trial was originally not powered to show the survival benefit. On the basis of these promising results, a Phase III randomized, placebo-controlled, double-blinded trial comparing LDAC with LDAC and volasertib in 660 patients (POLO-AML-2, NCT01721876) was initiated. Results are expected in early 2016.

As stated herein, Plk1 is an essential kinase in the cell cycle. Plk1 is overexpressed in a variety of neoplasms. Overexpression of Plk1 in tumors has been linked to poor outcomes. Volasertib is a specific inhibitor of Plk1; the question is whether volasertib is more effective in treating cancers that express higher levels of Plk1 than in treating those that express lower levels. The latest study ${ }^{77}$ using bone marrow biopsies from AML patients, leukemia cell lines, immortalized lymphoblastoid cell lines, and nonneoplastic cell lines treated with Plk1 inhibitor, however, showed uniform suppression of proliferation, followed by apoptosis regardless of Plk1 expression levels. Therefore, volasertib is more likely to offer an advantage for those patients whose uncontrolled cell proliferation in neoplasms poses the most serious threats to survival, eg, patients with AML having high leukocyte counts. The other side of the coin is that volasertib could cause some other unintended 
consequence if proliferation of normal cells is tightly suppressed too. It is difficult to link the cell proliferation status with efficacy in other tumor types at this time due to the small number and variety of neoplasms studied in these early-phase clinical trials.

\section{Conclusion}

AML assumes an aggressive course and is rapidly fatal if left untreated. It is unfortunately more common in the elderly population, with median age of diagnosis being above 65 years. Although most clinical trials show that elderly patients benefit from intensive treatment, it is likely that most patients in these trials comprise a very selective group of patients and it is unlikely that these studies are representative of the patients in the general population. This notion is supported by a recent SEER/Medicare analysis, which shows that $60 \%$ of patients older than 66 years of age were not offered any treatment. ${ }^{42}$ Despite intensive search for alternative, low-intensiveness regimens for the elderly and infirm patients unable to tolerate the standard treatment, the ideal regimen that adds benefit to single agent cytarabine proved to be elusive. In addition, the safety and tolerability of various chemotherapy drugs continues to be the biggest hindrance to the treatment of AML. Volasertib offers a newer approach to the treatment of AML. The promising results seen in a Phase II study with very favorable safety profile in both the AML studies and the solid tumor trials make this one of the exciting drugs in the pipeline for the treatment of AML. The Phase III volasertib trial results will, hopefully, provide an answer shortly.

\section{Acknowledgment}

The authors thank Lynsey Ekema, Master of Science Medical Illustration Instructional Design and Development, Georgia Regents University, for her expert assistance in illustrating the cell cycle in Figure 1.

\section{Disclosure}

The authors report no conflicts of interest in this work.

\section{References}

1. Ferrara F, Schiffer CA. Acute myeloid leukaemia in adults. Lancet. 2013;381(9865):484-495.

2. Estey E. Acute myeloid leukemia and myelodysplastic syndromes in older patients. J Clin Oncol. 2007;25(14):1908-1915.

3. Estey EH. Acute myeloid leukemia: 2013 update on risk-stratification and management. Am J Hematol. 2013;88(4):318-327.

4. Döhner H, Estey EH, Amadori S, et al; European LeukemiaNet. Diagnosis and management of acute myeloid leukemia in adults: recommendations from an international expert panel, on behalf of the European LeukemiaNet. Blood. 2010;115(3):453-474.
5. Grimwade D, Walker H, Oliver F, et al. The importance of diagnostic cytogenetics on outcome in AML: analysis of 1,612 patients entered into the MRC AML 10 trial. The Medical Research Council Adult and Children's Leukaemia Working Parties. Blood. 1998;92(7): 2322-2333.

6. Slovak ML, Kopecky KJ, Cassileth PA, et al. Karyotypic analysis predicts outcome of preremission and postremission therapy in adult acute myeloid leukemia: a Southwest Oncology Group/Eastern Cooperative Oncology Group Study. Blood. 2000;96(13):4075-4083.

7. Cancer Genome Atlas Research Network. Genomic and epigenomic landscapes of adult de novo acute myeloid leukemia. NEnglJ Med.2013; 368(22):2059-2074

8. Rowley JD. Chromosomal translocations: revisited yet again. Blood. 2008;112(6):2183-2189.

9. Döhner K, Paschka P. Intermediate-risk acute myeloid leukemia therapy: current and future. Hematology Am Soc Hematol Educ Program. 2014;2014(1):34-43.

10. Schnittger S, Kern W, Tschulik C, et al. Minimal residual disease levels assessed by NPM1 mutation-specific RQ-PCR provide important prognostic information in AML. Blood. 2009;114(11):2220-2231.

11. Alcalay M, Tiacci E, Bergomas R, et al. Acute myeloid leukemia bearing cytoplasmic nucleophosmin (NPMc + AML) shows a distinct gene expression profile characterized by up-regulation of genes involved in stem-cell maintenance. Blood. 2005;106(3):899-902.

12. Schlenk RF, Döhner K, Krauter J, et al; German-Austrian Acute Myeloid Leukemia Study Group. Mutations and treatment outcome in cytogenetically normal acute myeloid leukemia. $N$ Engl J Med. 2008; 358(18):1909-1918.

13. Ostronoff F, Othus M, Lazenby M, et al. Prognostic significance of NPM1 mutations in the absence of FLT3-internal tandem duplication in older patients with acute myeloid leukemia: a SWOG and UK National Cancer Research Institute/Medical Research Council Report. J Clin Oncol. 2015;33(10):1157-1164.

14. Breitenbuecher F, Schnittger S, Grundler R, et al. Identification of a novel type of ITD mutations located in nonjuxtamembrane domains of the FLT3 tyrosine kinase receptor. Blood. 2009;113(17):4074-4077.

15. Wander SA, Levis MJ, Fathi AT. The evolving role of FLT3 inhibitors in acute myeloid leukemia: quizartinib and beyond. Ther Adv Hematol. 2014;5(3):65-77.

16. Ley TJ, Ding L, Walter MJ, et al. DNMT3A mutations in acute myeloid leukemia. $N$ Engl J Med. 2010;363(25):2424-2433.

17. Gaidzik VI, Schlenk RF, Paschka P, et al. Clinical impact of DNMT3A mutations in younger adult patients with acute myeloid leukemia: results of the AML Study Group (AMLSG). Blood. 2013;121(23): 4769-4777.

18. Patel JP, Gönen M, Figueroa ME, et al. Prognostic relevance of integrated genetic profiling in acute myeloid leukemia. NEngl J Med. 2012; 366(12):1079-1089.

19. Popovici-Muller J, Saunders JO, Salituro FG, et al. Discovery of the first potent inhibitors of mutant IDH1 that lower tumor 2-HG in vivo. ACS Med Chem Lett. 2012;3(10):850-855.

20. Wang F, Travins J, DeLaBarre B, et al. Targeted inhibition of mutant IDH2 in leukemia cells induces cellular differentiation. Science. 2013; 340(6132):622-626.

21. Gaidzik VI, Paschka P, Späth D, et al. TET2 mutations in acute myeloid leukemia (AML): results from a comprehensive genetic and clinical analysis of the AML study group. J Clin Oncol. 2012;30(12):1350-1357.

22. Metzeler KH, Maharry K, Radmacher MD, et al. TET2 mutations improve the new European LeukemiaNet risk classification of acute myeloid leukemia: a Cancer and Leukemia Group B study. J Clin Oncol. 2011; 29(10):1373-1381.

23. Appelbaum FR. Haematological cancer: the rule of three in AML induction - is cladribine the answer? Nat Rev Clin Oncol. 2012;9(7): 376-377.

24. Bishop JF, Matthews JP, Young GA, et al. A randomized study of high-dose cytarabine in induction in acute myeloid leukemia. Blood. 1996;87(5):1710-1717. 
25. Wiernik PH, Banks PL, Case DC Jr, et al. Cytarabine plus idarubicin or daunorubicin as induction and consolidation therapy for previously untreated adult patients with acute myeloid leukemia. Blood. 1992; 79(2):313-319.

26. Fernandez HF, Sun Z, Yao X, et al. Anthracycline dose intensification in acute myeloid leukemia. N Engl J Med. 2009;361(13):1249-1259.

27. Löwenberg B, Ossenkoppele GJ, van Putten W, et al; Dutch-Belgian Cooperative Trial Group for Hemato-Oncology (HOVON); German AML Study Group (AMLSG); Swiss Group for Clinical Cancer Research (SAKK) Collaborative Group. High-dose daunorubicin in older patients with acute myeloid leukemia. N Engl J Med. 2009;361(13): $1235-1248$

28. Li W, Gong X, Sun M, et al. High-dose cytarabine in acute myeloid leukemia treatment: a systematic review and meta-analysis. PLoS One. 2014;9(10):e110153.

29. Anderson JE, Kopecky KJ, Willman CL, et al. Outcome after induction chemotherapy for older patients with acute myeloid leukemia is not improved with mitoxantrone and etoposide compared to cytarabine and daunorubicin: a Southwest Oncology Group study. Blood. 2002;100(12):3869-3876.

30. Goldstone AH, Burnett AK, Wheatley K, et al; Medical Research Council Adult Leukemia Working Party. Attempts to improve treatment outcomes in acute myeloid leukemia (AML) in older patients: the results of the United Kingdom Medical Research Council AML11 trial. Blood. 2001;98(5):1302-1311.

31. Rowe JM, Neuberg D, Friedenberg W, et al; Eastern Cooperative Oncology. A phase 3 study of three induction regimens and of priming with GM-CSF in older adults with acute myeloid leukemia: a trial by the Eastern Cooperative Oncology Group. Blood. 2004;103(2):479-485.

32. Giles FJ, Borthakur G, Ravandi F, et al. The haematopoietic cell transplantation comorbidity index score is predictive of early death and survival in patients over 60 years of age receiving induction therapy for acute myeloid leukaemia. Br J Haematol. 2007;136(4):624-627.

33. Walter RB, Othus M, Borthakur G, et al. Prediction of early death after induction therapy for newly diagnosed acute myeloid leukemia with pretreatment risk scores: a novel paradigm for treatment assignment. J Clin Oncol. 2011;29(33):4417-4423.

34. Grimwade D, Walker H, Harrison G, et al; Medical Research Council Adult Leukemia Working Party. The predictive value of hierarchical cytogenetic classification in older adults with acute myeloid leukemia (AML): analysis of 1065 patients entered into the United Kingdom Medical Research Council AML11 trial. Blood. 2001;98(5): $1312-1320$

35. Rowe JM. Consolidation therapy: what should be the standard of care? Best Pract Res Clin Haematol. 2008;21(1):53-60.

36. Bloomfield CD, Lawrence D, Byrd JC, et al. Frequency of prolonged remission duration after high-dose cytarabine intensification in acute myeloid leukemia varies by cytogenetic subtype. Cancer Res. 1998;58(18):4173-4179.

37. Burnett AK, Hills RK, Milligan DW, et al. Attempts to optimize induction and consolidation treatment in acute myeloid leukemia: results of the MRC AML12 trial. J Clin Oncol. 2010;28(4):586-595.

38. Gardin C, Turlure P, Fagot T, et al. Postremission treatment of elderly patients with acute myeloid leukemia in first complete remission after intensive induction chemotherapy: results of the multicenter randomized Acute Leukemia French Association (ALFA) 9803 trial. Blood. 2007;109(12):5129-5135.

39. Sorror ML, Maris MB, Storer B, et al. Comparing morbidity and mortality of HLA-matched unrelated donor hematopoietic cell transplantation after nonmyeloablative and myeloablative conditioning: influence of pretransplantation comorbidities. Blood. 2004;104(4):961-968.

40. Appelbaum FR, Gundacker H, Head DR, et al. Age and acute myeloid leukemia. Blood. 2006;107(9):3481-3485.

41. Mengis C, Aebi S, Tobler A, Dahler W, Fey MF. Assessment of differences in patient populations selected for excluded from participation in clinical phase III acute myelogenous leukemia trials. J Clin Oncol. 2003;21(21):3933-3939.
42. Medeiros BC, Satram-Hoang S, Hurst D, Hoang KQ, Momin F, Reyes C. Big data analysis of treatment patterns and outcomes among elderly acute myeloid leukemia patients in the United States. Ann Hematol. 2015;94(7):1127-1138.

43. Burnett AK, Milligan D, Prentice AG, et al. A comparison of low-dose cytarabine and hydroxyurea with or without all-trans retinoic acid for acute myeloid leukemia and high-risk myelodysplastic syndrome in patients not considered fit for intensive treatment. Cancer. 2007; 109(6):1114-1124.

44. Kayser S, Zucknick M, Döhner K, et al; German-Austrian AML Study Group. Monosomal karyotype in adult acute myeloid leukemia: prognostic impact and outcome after different treatment strategies. Blood. 2012;119(2):551-558.

45. Kantarjian HM, Thomas XG, Dmoszynska A, et al. Multicenter, randomized, open-label, phase III trial of decitabine versus patient choice, with physician advice, of either supportive care or low-dose cytarabine for the treatment of older patients with newly diagnosed acute myeloid leukemia. J Clin Oncol. 2012;30(21):2670-2677.

46. Montalban-Bravo G, Garcia-Manero G. Novel drugs for older patients with acute myeloid leukemia. Leukemia. 2015;29(4):760-769.

47. Petersdorf SH, Kopecky KJ, Slovak M, et al. A phase 3 study of gemtuzumab ozogamicin during induction and postconsolidation therapy in younger patients with acute myeloid leukemia. Blood. 2013; 121(24):4854-4860.

48. Amadori S, Suciu S, Selleslag D, et al. Randomized trial of two schedules of low-dose gemtuzumab ozogamicin as induction monotherapy for newly diagnosed acute myeloid leukaemia in older patients not considered candidates for intensive chemotherapy. A phase II study of the EORTC and GIMEMA leukaemia groups (AML-19). Br J Haematol. 2010;149(3):376-382.

49. Burnett AK, Hills RK, Hunter AE, et al; UK National Cancer Research Institute AML Working Group. The addition of gemtuzumab ozogamicin to low-dose Ara-C improves remission rate but does not significantly prolong survival in older patients with acute myeloid leukaemia: results from the LRF AML14 and NCRI AML16 pick-a-winner comparison. Leukemia. 2013;27(1):75-81.

50. Strebhardt K. Multifaceted polo-like kinases: drug targets and antitargets for cancer therapy. Nat Rev Drug Discov. 2010;9(8):643-660.

51. Lens SM, Voest EE, Medema RH. Shared and separate functions of polo-like kinases and aurora kinases in cancer. Nat Rev Cancer. 2010; 10(12):825-841.

52. Petronczki M, Lenart P, Peters JM. Polo on the rise-from mitotic entry to cytokinesis with Plk1. Dev Cell. 2008;14(5):646-659.

53. Llamazares S, Moreira A, Tavares A, et al. Polo encodes a protein kinase homolog required for mitosis in Drosophila. Genes Dev. 1991; 5(12A):2153-2165.

54. Clay FJ, McEwen SJ, Bertoncello I, Wilks AF, Dunn AR. Identification and cloning of a protein kinase-encoding mouse gene, Plk, related to the polo gene of Drosophila. Proc Natl Acad Sci U S A. 1993; 90(11):4882-4886.

55. Hamanaka R, Maloid S, Smith MR, O'Connell CD, Longo DL, Ferris DK. Cloning and characterization of human and murine homologues of the Drosophila polo serine-threonine kinase. Cell Growth Differ. 1994;5(3):249-257.

56. Santamaria A, Neef R, Eberspächer U, et al. Use of the novel Plk1 inhibitor ZK-thiazolidinone to elucidate functions of Plk1 in early and late stages of mitosis. Mol Biol Cell. 2007;18(10):4024-4036.

57. Leung GC, Hudson JW, Kozarova A, Davidson A, Dennis JW, Sicheri F. The Sak polo-box comprises a structural domain sufficient for mitotic subcellular localization. Nat Struct Biol. 2002;9(10):719-724.

58. Barr FA, Sillje HH, Nigg EA. Polo-like kinases and the orchestration of cell division. Nat Rev Mol Cell Biol. 2004;5(6):429-440.

59. Song B, Liu XS, Liu X. Polo-like kinase 1 (Plk1): an unexpected player in DNA replication. Cell Div. 2012;7:3.

60. Smits VA, Klompmaker R, Arnaud L, Rijksen G, Nigg EA, Medema RH. Polo-like kinase-1 is a target of the DNA damage checkpoint. Nat Cell Biol. 2000;2(9):672-676. 
61. van Vugt MA, Smits VA, Klompmaker R, Medema RH. Inhibition of polo-like kinase-1 by DNA damage occurs in an ATM- or ATRdependent fashion. J Biol Chem. 2001;276(45):41656-41660.

62. van Vugt MA, Bras A, Medema RH. Polo-like kinase-1 controls recovery from a G2 DNA damage-induced arrest in mammalian cells. Mol Cell. 2004;15(5):799-811.

63. Liu X, Erikson RL. Polo-like kinase (Plk)1 depletion induces apoptosis in cancer cells. Proc Natl Acad Sci U S A. 2003;100(10):5789-5794.

64. Tamura Y, Simizu S, Muroi M, et al. Polo-like kinase 1 phosphorylates and regulates $\mathrm{Bcl}-\mathrm{x}(\mathrm{L})$ during pironetin-induced apoptosis. Oncogene. 2009;28(1):107-116.

65. Rizki A, Mott JD, Bissell MJ. Polo-like kinase 1 is involved in invasion through extracellular matrix. Cancer Res. 2007;67(23):11106-11110.

66. Rudolph D, Steegmaier M, Hoffmann M, et al. BI 6727, a polo-like kinase inhibitor with improved pharmacokinetic profile and broad antitumor activity. Clin Cancer Res. 2009;15(9):3094-3102.

67. Lénárt P, Petronczki M, Steegmaier M, et al. The small-molecule inhibitor BI 2536 reveals novel insights into mitotic roles of polo-like kinase 1. Curr Biol. 2007;17(4):304-315.

68. Winkles JA, Alberts GF. Differential regulation of polo-like kinase 1, 2, 3, and 4 gene expression in mammalian cells and tissues. Oncogene. 2005;24(2):260-266.

69. Takai N, Hamanaka R, Yoshimatsu J, Miyakawa I. Polo-like kinases (Plks) and cancer. Oncogene. 2005;24(2):287-291.

70. Weiss L, Efferth T. Polo-like kinase 1 as target for cancer therapy. Exp Hematol Oncol. 2012;1(1):38.
71. Renner AG, Dos Santos C, Recher C, et al. Polo-like kinase 1 is overexpressed in acute myeloid leukemia and its inhibition preferentially targets the proliferation of leukemic cells. Blood. 2009;114(3):659-662.

72. Steegmaier M, Hoffmann M, Baum A, et al. BI 2536, a potent and selective inhibitor of polo-like kinase 1 , inhibits tumor growth in vivo. Curr Biol. 2007;17(4):316-322.

73. Gjertsen BT, Schoffski P. Discovery and development of the polo-like kinase inhibitor volasertib in cancer therapy. Leukemia. 2015;29(1):11-19.

74. Döhner H, Lübbert M, Fiedler W, et al. Randomized, phase 2 trial of low-dose cytarabine with or without volasertib in AML patients not suitable for induction therapy. Blood. 2014;124(9):1426-1433.

75. Schöffski P, Awada A, Dumez H, et al. A phase I, dose-escalation study of the novel polo-like kinase inhibitor volasertib (BI 6727) in patients with advanced solid tumours. Eur J Cancer. 2012;48(2):179-186.

76. Lin CC, Su WC, Yen CJ, et al. A phase I study of two dosing schedules of volasertib (BI 6727), an intravenous polo-like kinase inhibitor, in patients with advanced solid malignancies. Br J Cancer. 2014;110(10):2434-2440.

77. Münch C, Dragoi D, Frey AV, et al. Therapeutic polo-like kinase 1 inhibition results in mitotic arrest and subsequent cell death of blasts in the bone marrow of AML patients and has similar effects in nonneoplastic cell lines. Leuk Res. 2015;39(4):462-470.
OncoTargets and Therapy

\section{Publish your work in this journal}

OncoTargets and Therapy is an international, peer-reviewed, open access journal focusing on the pathological basis of all cancers, potential targets for therapy and treatment protocols employed to improve the management of cancer patients. The journal also focuses on the impact of management programs and new therapeutic agents and protocols on

\section{Dovepress}

patient perspectives such as quality of life, adherence and satisfaction. The manuscript management system is completely online and includes a very quick and fair peer-review system, which is all easy to use. Visit http://www.dovepress.com/testimonials.php to read real quotes from published authors. 